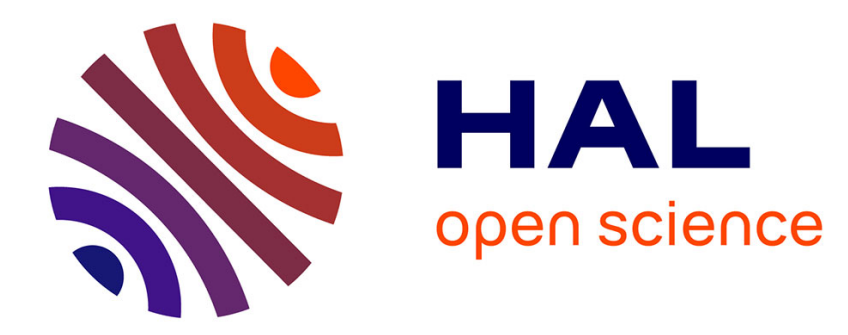

\title{
"CHEMICAL" REACTIVITY ON THE DISORDERED SURFACES. THE CASE OF ICE
}

\author{
J. Ocampo, B. Schmitt, J. Klinger
}

\section{To cite this version:}

J. Ocampo, B. Schmitt, J. Klinger. "CHEMICAL" REACTIVITY ON THE DISORDERED SURFACES. THE CASE OF ICE. Journal de Physique Colloques, 1987, 48 (C1), pp.C1-557-C1-563. 10.1051/jphyscol:1987176 . jpa-00226323

\section{HAL Id: jpa-00226323 https://hal.science/jpa-00226323}

Submitted on 1 Jan 1987

HAL is a multi-disciplinary open access archive for the deposit and dissemination of scientific research documents, whether they are published or not. The documents may come from teaching and research institutions in France or abroad, or from public or private research centers.
L'archive ouverte pluridisciplinaire HAL, est destinée au dépôt et à la diffusion de documents scientifiques de niveau recherche, publiés ou non, émanant des établissements d'enseignement et de recherche français ou étrangers, des laboratoires publics ou privés. 
"CHEMICAL" REACTIVITY ON THE DISORDERED SURFACES. THE CASE OF ICE

\author{
J. OCAMPO, B. SCHMITT and J. KLINGER
}

Laboratoire de Glaciologie et Géophysique de I'Environnement, BP 96, F-38402 st-Martin-d'Hères Cedex, France

\begin{abstract}
RESUNE : La cinetique de clathration du dioxyde de carbone et du krypton sur la surface de la glace a été etudiée. Afin de comprendre le rôle joué par une surface desordonnée sur la clathration la "rugosité" de la surface est caracterisée par sa dimension fractale d. Cette dimension a été calculée de manière indirecte à partir de la diffusion des molecules d'eau pendant le fritage des particules de glace et pendant la clathration. Le fait que $\widetilde{\mathrm{d}}<2$ implique que l'exploration est "compacte"ce qui influence fortement le fritage et la clathration.

ABSTRACT : The kinetics of krypton and carbon dioxyde clathration on ice surface was studied. In order to understand the role played by a disordered surface in clathration, the surface"roughness" was characterized by the spectral dimension d". This dimension was calculated indirectly from the water diffusion during the sintering and clathration of ice particles. Since $\tilde{d}<2$ the exploration is "compact " which strongly influences the sintering and clathration.
\end{abstract}

\title{
INTRODUCTION
}

The clathration by gas-ice reaction has been observed since 1962 [ 1 to 4]. Barrer and Edge theoretical approach was unique and based on. the concept of preexisting cages being filled as Langmuir isotherms.

This paper atterpts to apply the physics of fractal structures to the measure of the degree of surface disorder and the clathration kinetics.

During these past few years, the concept of fractal structures developed by Mandelbrot [5] has been of great utility for the description of structures with geometrical and dynamical properties which appear purely random [6]. Avnix and Farin [7] have remarked that the surfaces of most materials are fractals at the molecular scale. Since it is difficult to oonceive any dynamic interfacial process that is independent of surface irregularities and defects, the study of gas clathration kinetics on the ice surface seems obviously very important. Catalysis is a phenomenon governed by such surface characteristics and certainly plays an important role in clathration.

\section{EXPERIMENTAL PROCEDURES}

The ice powder sample was prepared by deionized water condensation(18 M// $25^{\circ} \mathrm{C}$ ) at $77 \mathrm{~K}$. Amorphous ice is obtained. After heating the sample up to $200 \mathrm{~K}$ amor- 
phous ice is transformed into hexagonal ice. From single crystal we have also prepared a powder ice after crushing the ice in vacoum at $77 \mathrm{~K}$.

We observed the evolution of specific surface area and heat of adsorption of the powder ice annealed at 195K, by means of krypton adsorption isotherms at $77 \mathrm{~K}$. The classical mancmetric method used is described elsewhere[8]. From this evolution, we have characterized the initial surface by a spectral dimension.

The formation of krypton and carbon dioxyde clathrates was done at 195K and monitored in the same way as an adsorption isotherm [9]. The krypton clathration is possible only if the surface contains the germ of another clathrate. The equilibrium pressure was compared to data in the Ilterature(usually obtained from clathrates prepared by freezing of a gas liquid mixture). To confirm the formation of clathrates, the surface reaction was followed qualitatively with an X-ray diffractometer.

\section{RESULTS}

Figure 1 shows the evolution of the specific surface area and heat of adsorption for crushed ice measured with krypton at 77K. The sample was annealed at $195 \mathrm{~K}$ for different time intervals and came back to $77 \mathrm{~K}$ to avoid evolution and to measure the krypton isotherms.

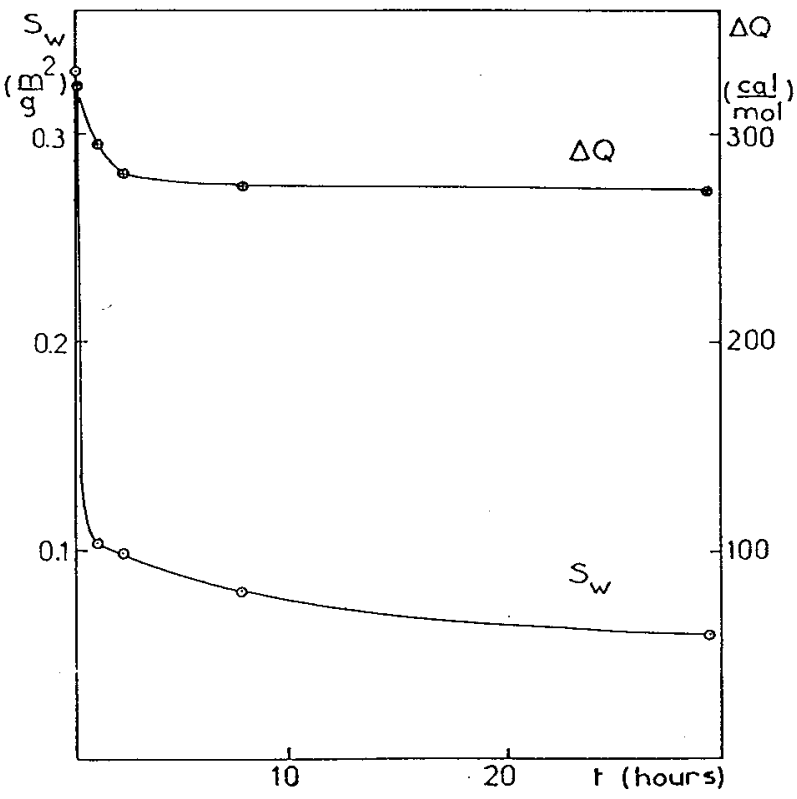

Figure 1: Specific surface area $\left(S_{w}\right)$ and adsorption heat $(\Delta Q)$ evolution. Krypton was used to measure adsorption isotherms at $77 \mathrm{~K}$ between differents annealing periods at $195 \mathrm{~K}$.

We can observe a fast evolution of the specific surface area and heat of adsorption in the first hour of the annealing period. The specific surface area decreased from $0,33 \mathrm{~m}^{2} / \mathrm{g}$ (fresh ice surface)to $0,06 \mathrm{~m}^{2} / \mathrm{g}$, close to the equilibrium value observed after 30 hours of annealing. 
The krypton clathration was done when the heat of adsorption equilibrium was fully attained and after clathration and decomposition with air. A gem of air clathrate is suspected to exist after this decomposition and to serve as a nuclel for the kryptan clathration.

In the case of carbon dioxyde, the heat of adsorption is constant for several hours. The incubation time seems linked with this phenomenon.

Figure 2 and 3 show the evolution of carbon dioxyde and krypton clathration. No incu bation time is observed for krypton. The equilibrium pressure at 195K for the clathrates was 253 mbar and 280 mbar for krypton and carbon dioxyde respectively.

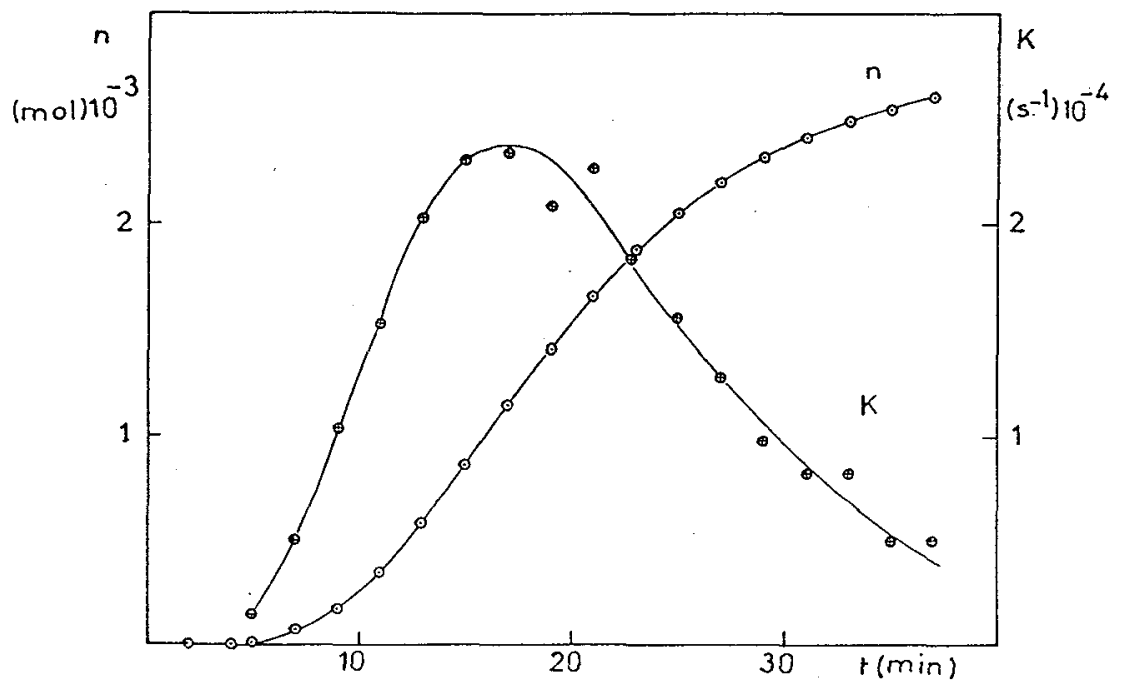

Figure 2 : Carbon dioxyde clathration $(n)$ and evolution of the reaction rate constant $(K)$ at $195 K$.

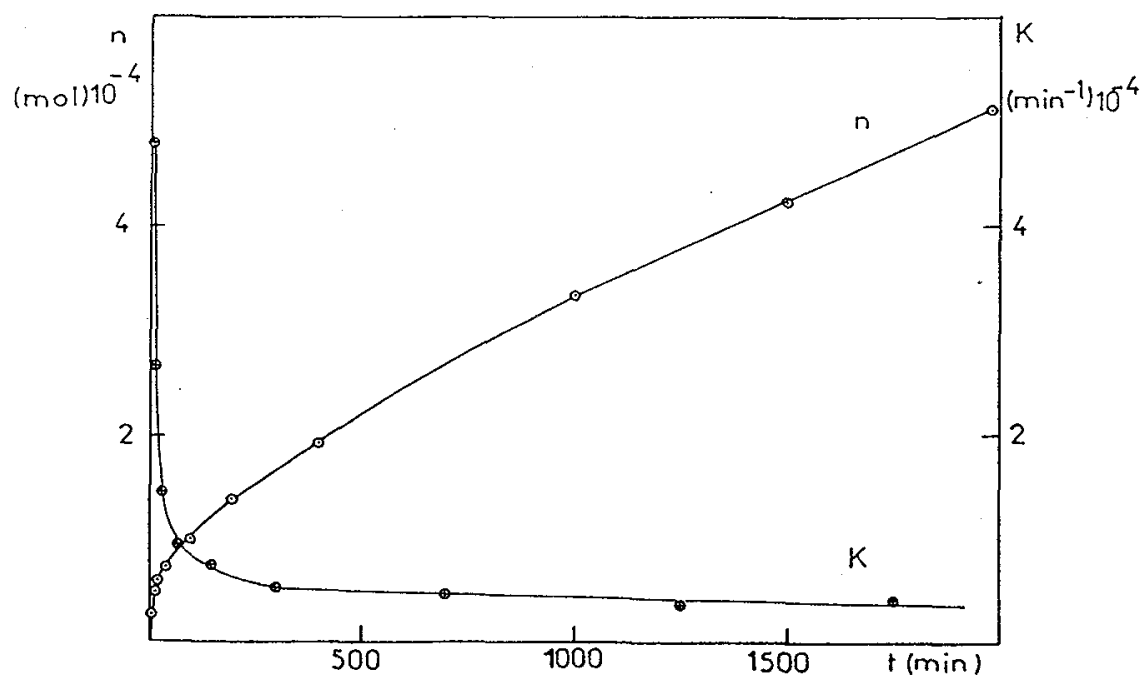

Figure 3 : Krypton clathration $(n)$ and evolution of the reaction rate constant (K) at $195 \mathrm{~K}$. 
The reaction rates constants shown in these figures were calculated from pressure $P$ evolution towards the equilibrium pressure $P_{\text {eq }}$ by the following equation :

$$
K=(\mathrm{dP} / \mathrm{dt}) /\left(\mathrm{P}-\mathrm{P}_{\mathrm{eq}}\right)
$$

We observe a time dependent behaviour of the reaction rate constant for both krypton and carbon dioxyde, even far from the saturation.

\section{DISOUSSION}

The essential features of our results may be interpreted in the following way: Our sample preparation method creats a great number of surface defects which are randomly distributed, therefore making a soft surface. The instability of both broken (or non compensated) hydrogen bonds and high surface enexgy probably causes the evolution of the specific surface area. This evolution seems to take place in a "sintering" fashion probably by short range migration of diffusing elements as we will see below.

The average number of "sintering or fusion sites" in a fractal space which have been visited by a random walker from the start until the time $t$, before its capture is [1]):

$$
N(t)=N_{0} t^{\tilde{d} / 2}
$$

where $\tilde{d}$ is the fracton(or spectral) dimension.

The differential equation which governs the chemical reaction between two molecules $A$ and $B$ is :

$$
-\dot{n}_{A}=-\dot{n}_{B}=K n_{A} n_{B}
$$

where $\mathbf{n}$ is the concentration before capture and

$$
K=K_{0} N(t) / t
$$

When fusion occurs : $n_{A}=n_{B}=A S_{W}$ (proportional to specific surface area). Then, from equations (1) to (3) we obtain :

$$
s_{w}=\left(\tilde{d} t^{-\tilde{d} / 2}\right) /\left(2 k_{0} N_{0} A\right)
$$

and from the graph slope of $\log s$ vs $\log t$ we found $: \tilde{d}=0.37$.

It means that the exploration in this space is "compact" ( $\tilde{d}<2)[11])$ in the sense that it strongly influences the capture laws. If our system is considered as a percolation one [6] the fractal dimension would be $: \bar{d}=0.52$, which satisfies the relation : $\mathrm{d}>\overline{\mathrm{d}}>\widetilde{\mathrm{d}}$.

The value of 0.52 confirms our hypothesis of "adhesion points" existence where the sintering takes place in short range diffusion paths.

In order to identify the nature of diffusing elements, we have estimated the activation energies of the rates $:\left(d S_{w} / d t\right)$ and $(d \Delta Q / d t)$ as the average rates at the begining of the evolution.

The rate of decreasing surface area has an activation energy of $E_{w}=-0.05 \mathrm{eV}$. for temperatures lower than $213 \mathrm{~K}$ and the rate of decreasing adsorption heat has an activation energy of $\mathrm{E}_{\mathrm{a}}=-0.16 \mathrm{eV}$ for temperatures lower than $258 \mathrm{~K}$. These energies are probably due to defect migration on the ice surface (as $O H$ or self interstitials [12][13]). The adsorption decrease was already seen in this range of temperatures and is probably due to the creation of a "proton surface" [14]. 
At temperatures near the melting point of ice, the rate of decreasing adsorption heat has an activation enengy of $E_{S}=1.12 \mathrm{eV}$ which is similar to the surface selfdiffusion energy.

The carbon dioxyde clathration at $195 \mathrm{~K}$ starts after an incubation period during which there is no capture.At this time, the number of captured molecules grows slowly. Fran the beginning gemination a self-catalytic process maintains the reaction. About the time dependent rate constent $K$ for krypton and carbon dicoryde we note that this anomalous reaction law depends on the "cleaness" of the surface (absence of germ), the host molecule nature and the emui]ibrium nressure. The surface where clathration cocurs is certainily very disordered and we can verify its fractal character in the same way as we did for the specific surface area.

From the differential equation (2) and if we consider that : $n_{A}<n_{B}$ (supertrapping process and far from the saturation), we can have a solution :

$$
n_{A}=n_{A}^{0} \exp \left(-2 k_{0} n_{B}^{0} t^{\tilde{d} / 2}, \tilde{d}\right)
$$

In our case of clathration, the pressure is proportional to the concentration in the gas phase : $n_{A}=\alpha$ P.Moreover, the water moleculeconcentration $\left(n_{B}=n_{\text {water }}\right)$ is con sidered as an infinite reservoir. Then from :

$$
\log (|\mathrm{P}| / \mathrm{p})=\log k_{0} n_{B}^{0}+\left(\frac{\tilde{d}}{2}-1\right) \log t
$$

we found for krypton clathration : $\tilde{d}=1.28$. For a penoolation system, the fractal dimension would be : $\bar{d}=1.79$.

Then the reaction for the krypton clathration seems to $_{2}$ occur in quasi-linear paths. The diffusion constant estimated is : $D_{\mathrm{kr}}=3 \cdot 10^{-13} \mathrm{~cm} / \mathrm{s}$.

In the case of carbon dioxyde clathration, frollowing the incubation period we can observe a first onder reaction with a diffusion constant and an activation energy of:

$$
D_{s}=10^{-9} \mathrm{~cm}^{2} / \mathrm{s} \quad E_{s}=-1.13 \mathrm{eV}
$$

After this evolution the reaction rate decreases as a zero onder reaction with a diffusion constant and activation enrrgy of:

$$
D_{v}=10^{-14} \mathrm{~cm}^{2} / \mathrm{s} \quad E_{v}=-0.7 \mathrm{ev}
$$

Those values which are very similar to surface self-diffusion and bulk selfdiffusion of water molecules in the ondinary hexagonal ice show that the diffusive element seems to be the water molecule.

The phase transformation from hexagonal to cubic clathrate structure is then started by the capture of the adsorbed gas molecule by water molecules migration . For krypton it was necesary to have a residual clathrate of another gas in order to start the clathration. The reaction constant attains a constant value after scme time and illustrates a transition from fractal kinetics to classical ones.

\section{DONCLUSION}

The ice powder surface was considered as a fractal space and then characterized by a spectral dimension. A kinetics criterion, based on adsorption sites diminution and clathration was used to calculate the spectral dimension.

The ice particles "punctual sintering" seems to ocour in a space where the spectral dimension is : $\tilde{\mathrm{d}}=0.37$, probably by surface defects migration in order to compensate the broken: hydrogen boinds and to diminish the surface energy. 
On the other hand the capture of gas molecules in clathration seems to occur in a fractal space where the spectral dimension is $\widetilde{d}=1.28$; The diffusion on ice surface is realized by the water molecules and respects a quasi-linear paths. The apparent bulk diffusion of the gas molecules is possibly tue to existing free cages in the clathrate structure and to bulk self-diffusion of water molecules.

The natural occurrence of gas hydrates has been mentioned in the past.Miller[15] has cormented their possible occurrence in the solar system. Caxbon dioxyde hydrate seens to exist on Mars and in comets. The first reported natural occurrence of clathrate hydrates on the Earth is the air hydrate in Antarctic foe-cores [16]. Glaciological and astrophysical applications of this study are described elsewhere[17].

\section{ACANOWLEDGEMENTS}

Financial support by the "Institut Françats des Sciences de 1 'Univens" ATP grant $\mathrm{N}^{\circ}$ 5724 is gratefully acknowledged.

\section{REFERENCES}

[1] BARRER, R. M., RUSICKA, D. J., Trans. Faraday SOc. 58 (1962b) 2262-2271.

[2] BARRER, R:. M., EDGE, A. V. J., PNOC. ROY. SOC. A300 (1967) 1-24.

[3] OREM, M. W., ADAMSOM, A. W., J. Collaid Sai. 31 (1969) 278-286.

[4] ADAMSOM, A. W., Javes, B. R., J. Colloid Sci. 37 (1971) 831-835.

[5] MANDEIBBROT, B. B., "Les objets fractals: fome, hasard et dimension" (Flamarion) 1975.

[6] ORBACH, R., Science, 231 (1986) 814-819.

[7] AVNIR, D., FARIN, D., Nature, 308 (1984) 261-263.

[8] OCAMPO, J., KLINGER, J., J. Colloid Sci. 86 (1982) 377-383.

[9] OCAMPO, J., KLINGER, J., J. Phys. Chem. 87 (1983) 4167-4170.

[10] ALEXANDER, S., ORBACH, R., J. Physique Lett. 43 (1982) L- 625-631.

[11] DE GENNES, P. G., C. R. Hebd. Séan. Acad. Sci. 296 (1983) Serie II, 881-885

[12] VON HIPPEL, A., J. Chem. Ptyys. 54 (1971) 145.

[13] GOTO, K., HONDO, T., HIGASHI, A., Jpn. J. Appl. PhYs. 25 (1986) 351-357.

[14] FLETCHER, N. H., Ed., E. Whalley, S. J. Jones and L. W. Gold Royal society of Canada, Otawa (1973) 132-136.

[15] MILLER, S., "Ices in the solar sytem", Klinger, Benest, Dollfus, Smoluchowski ed. (Reidel Publishing Co.) 1985.

[16] MrLLER, S., Ed., E. Whalley, S. J. Jones and L. W. Gold Royal Society of Canada, Otawa (1973) 42-50.

[17] OCAMPO, J., "Adsonption, clathration of gas molecules and mobility of water molecules on the ice interfaces. Glaciological and astrophysical applications "Thése d'Etat, Université Scientifique et Medicaie de Grenoble, France (1986).

\section{COMMENTS}

\section{P.L.M. PLUMMER}

Have you done your fractal analysis in higher dimensions and if so were your results similar as to the fractal dimensions of the process? If you have not carried out the analysis would you care to speculate on the results? 
Answer :

No. At lower terperatures and in microporous ice we will have may be a fractal dimension between 2 and 3 .

J.P. DEVLIN

We find that polar molecules (such as ethylene oxide) are much more effective in inducing clathrate hydrate formation. Do you have any comparative rate data for polar vs. nonpolar molecules.

Answer : no.

\section{J.S. TSE}

What was the gas pressure in your adsorption experiment ?

Answer :

For $\mathrm{CO}_{2}$ at $195 \mathrm{~K}$ not higher than one bar. Always in the phase diagram region for clathrate formation. 